\title{
Bohr Correspondence Principle and Multiphoton Nature Raleigh Light Scattering
}

\author{
Valeriy E. Ogluzdin \\ Prokhorovs General Physics Institute, Russian Academy of Sciencesul, Moscow, Russia \\ E-mail: ogluzdin@kapella.gpi.ru \\ Received January 20, 2010; revised April 6, 2010; accepted April 10, 2010
}

\begin{abstract}
The correspondence principle and the condition of supplementation were introduced by N. Bohr for the submission of light phenomena, taking into account the wave nature of electromagnetic radiation on one hand, and its quantum structures on the other. In this paper, correspondence principle combines two models of matter, namely, the classical point of view of environment can be considered as an ensemble no equally-frequencies oscillators, i.e. electrons in the surrounding various atoms (molecules) of the matter and characterized by its own set of frequencies (but not hesitant in the absence of an energy source) and the quantum - environment could be presented as a set (ensemble) two-level systems, a wide range of Bohr frequencies. According to the correspondence principle Bohr jump-frequencies of atoms (molecules or nano particles) and natural frequencies oscillations of electrons of the same environment - oscillators are equal to each other. The dispersion characteristics of the environment in the every study range of optical frequencies correspond to the model of the classical harmonic oscillator of Lorenz, capable oscillates with Bohr frequency. Using the laws of classical mechanics to describe the environment and its dispersion properties, and the simultaneous presentation of light radiation in the form of a beam interacting with the environment of photons (quanta, corpuscles) helps explain peculiarities of the spectral composition Raleigh light scattered.
\end{abstract}

Keywords: Correspondence Principle, Complementarily Condition, Raleigh Light Scattering, Classical Harmonic Oscillator of Lorenz, Bohr Jump-Frequencies

\section{Experimental Research RLS in Organic Liquids by Fabelinskiy I. L. (1957)}

The purpose of this message - the description of multiphoton model of Raleigh light scattering (RLS), using the correspondence principle of N. Bohr.

Before turning to interpret RLS through multiphoton model, we should remember the results of early experiments, performed by I. L. Fabelinskiy (1957) [1], in which it was found, that the fine structure of the spectrum of monochromatic light, scattered in pure organic liquids, as a rule, consists of three spectral components. Recall that to observe RLS in these experiments as a source of radiation is used commonly available for such studies mercury low pressure spectral lamp (line $\lambda=$ $4358 \mathrm{~A}^{\circ}$ ). Through prolonged exposure photo plates could benefit from economies of accumulation, which even in the case of a weak signal was guaranteed for black photo recording. In the article was presented an overview of theories of molecular scattering of light in pure liquids as well as a list of experimental works on this subject (before 1957).

Typically, the structure of the spectrum RLS of pure organic liquid contains three spectral components. The spectral shifts Stocks and anti-Stocks components concerning of the central, unbiased, the most glaring components usually are not equal to one another. In toluene, benzene and $\mathrm{CS}_{2}$ shifts Stocks and anti-Stocks components of the triplet in different series of experiments for each substance do not coincide with each other. This asymmetry was noted by I. L. Fabelinskiy, and also attention was drawn to the fact that anti-Stocks line less intense than Stocks. According to I. L. Fabelinskiy, is the real reason for asymmetry shifts Stokes and anti-Stocks components remained unknown while writing the work? Disagreement Stocks and anti-Stocks components on the frequency of the incident radiation may indicate that the emergence of the spread of radiation components are not connected to each other and are independent of each other.

This difference is due to excitation of independent os- 
cillators in the environment, some oscillator's environment is responsible for Stocks frequency components, while others-for anti-Stocks; detection of natural frequencies of these oscillators and their search-is our goal.

The only simultaneous recognition dispersion characteristics of refractive indexes in this spectral field of different numerous (equal and unequal frequencies) oscillators $[2,3]$, uniformly filling the entire volume of the environment can provide an answer to the question of different intensity Stocks, anti-Stocks components of the fine structure of RLS.

\section{Correspondence Principle of N. Bohr [4]; Bohr Jump-Frequencies [5,6].}

In the article, in the future statement considerable attention will be given to the simple oscillators of the medium. It is their presence in the environment can be attributed to our attention to the correspondence principle and to the complementarily condition N. Bohr. The correspondence principle and the complementarily condition has been proposed by N. Bohr (1932) in order to remain as long as possible in terms of the concepts of classical physics, and as long as possible to explain physical regularities with simple graphic models. Recall that the correspondence principle (principle of supplementary, complementarily, subsidiary) has been used Bohr to explain the relationship between the electromagnetic field and light quanta [4].

In such system we have in accordance with the principle of correspondence (supplementary, complementarily, subsidiary) different oscillators of environment associated with the electrons among the various two-level formations (with atomic skeletons, fragments of molecules, nanoparticles), the distance between the levels which can be uniquely represented through the Bohr jump-frequency $v_{0 \mathrm{i}}$ (see Fermi E. 1965) [5]:

$$
v_{0 \mathrm{i}}=\left(E_{\mathrm{i}}-E_{0}\right) / h
$$

here $E_{\mathrm{i}}, E_{0}$-energy of excited and ground levels of medium; $h$-Planck constant.

Important is the fact that the electrons in atomic (molecular ) environment can be represented in the form of classic oscillators, each depending on the position in the atom (molecule, nanoparticle) situation characterized by some intrinsic of frequency vibrations. The frequency $v_{0}$ i can be determined from the relationship between mass of the electron $m$ and the coefficient of elasticity $G_{\mathrm{j}}$, describing stiffness connection of electrons with the skeleton atom or molecule (Garbuny M. 1965) [7].

These frequencies (indices $i$ and $j$ denotes multiple frequencies and communications within the molecule or nanoparticle: $i=1,2,3 \ldots \infty ; j=1,2,3 \ldots \infty)$ easy to detect and identify if we have the absorption or radiation spectra of the investigated substance [6]. The degree of absorption (no transparency) of weak radiation by envi- ronment in different parts of the spectrum and there are ultimately a set of characteristic frequencies $v_{0 \mathrm{i}}$ for the given medium.

Narrow-lines nature of atomic spectra and stripe-ines of molecular evidence that in the case of atoms electron interaction with atomic skeleton (for the hydrogen - kernel) is more simple than in the case of molecules or different-sized nanoparticles consisting of the ensemble of molecules or atoms connected to one another.

At the same time, note that in the absence of a source of excitation (that is, spectral lamps, arc, solar radiation or laser) of investigated environment, its own characteristics (Bohr jump-frequencies) do not appear explicitly, and their detection is difficult.

As our research on scattering almost resonance radiation in atomic vapor metals [8,9] or photoluminescence phenomenon of $\mathrm{Si}$-powder in the ethanol $[10,11]$ in these cases, direct observation characteristic Bohr jump-frequencies $v_{0 i}$ environment with help of one-frequency $v$ laser is made difficult and it is necessary additional measurements spectrum. Their location can be calculated only on the basis of processing of spectrograms, using the ratio, follows from the conservation energy law, that takes into account the spectral characteristics of environment and has a kind of:

$$
v_{0 i}=2 v-v_{m}
$$

here $v$ is the frequency of the radiation, that affects the medium (environment); $v_{0 \mathrm{i}}$-frequencies oscillators environment, they are Bohr jump-frequencies; $h$ - Planck constant is omitted;

index $m$ is replaced by $s$ (stoks), if $v<v_{0 i}$,

index $m$ is replaced by as (anti-stokes), if $v>v_{0 i}$.

Indexes $s$ and as suited to the observed in the experiment Stocks and anti-Stocks components radiation, scattered by atomic medium [8,9], and can be applied to photoluminescence $[10,11]$.

If environment has continuous spectrum of absorption, then using harmonics Fourier decomposition, we can give all set of lines, each being determined her proper Bohr jump-frequency.

A few words about the relationship (2):

$$
v_{m}=2 v-v_{0 i}
$$

According to the theory [12] the probability of such processes is low. However, all may be significantly simplified, if one recalls the principle of correspondence and classic dispersion theory, which describes the behavior of the refractive index medium $n(v)$ near natural frequencies $v_{0 i}$ classic Lorenz harmonic oscillators. Since in this case the refractive index medium or less than unit, if $v>v_{0 i}$, or many more units, if $v<v_{0 i}$, the processes, described by relationship (3), are playing a decisive role in these areas spectra, and, consequently; in this time the the lower-order processes are ineffective. It is from this perspective, we try to understand the nature RLS and its complex spectral correct structure [1]. 


\section{Dispersion of the Refractive Index $N(v)$ of Environment, Consisting of a Classical Harmonic Oscillators Lorenz [2,3]; Multiphoton Nature RLS.}

The purpose of this work is to explain the nature RLS, based on attracting mechanisms suited to the role of characteristic frequency oscillators $v_{0 i}$ environment, as well as contribute to the scattering processes multiphoton interraction. Note that these characteristic frequencies oscillators $v_{0 i}$ we can compare (or equate them) Bohr frequencies and use the condition of complementarity of Bohr. The correspondence principle and condition of complementarity of Bohr in this case connect the conclusions follow from the model of classical harmonic oscillator of Lorenz, on the one hand, and, on the other hand, allow considering a two-level environments model and the consequences arising from this model. We are in this regard, in particular, would be interested in the correspondence between the states of the classical harmonic oscillator Lorenz and the electronic levels of atoms (molecules) of a two-level environment (in quantum model).

It should be noted, that in the classical model oscillator can be hesitate - after receiving a portion of energy, or be able to stop; and one quarter period vibration oscillators corresponds to a single act of converting kinetic energy into potential, or vice versa. Such a portion of energy in the quantum model of a two-level atomic system corresponds to the transition of electron from the level to level. This portion energy can either be absorbed or be emitted. Once again, we remind you that if power to the system is not introduced, the identification of frequencies $v_{0 \mathrm{i}}$, characterizing environment, difficult.

But in this case, we must remember that all frequencies $v_{0 i}$ environment, their full "virtual" spectrum is the calling card of this media. And all frequencies $v_{0 i}$-unique and independent performances of the environment.

Due to the introduction of classical oscillators naturally becomes our approach to the classical theory of dispersion of the refractive index $n(v)$ of environment, consisting of an ensemble of classical harmonic oscillators Lorenz. According to the theory of dispersion Lorenz $[2,3]$ for the frequency of the incident radiation is less than the natural frequency oscillators $v_{0 i}$, the refractive index of environment more unit and with the reduction of the difference between frequency of the incident radiation $v$ and frequency oscillators $v_{0 i}$ the refractive index $n(v)$ can grow indefinitely: $n(v) \gg 1$ [2].

Experiments on slow light, performed most recently perfectly illustrate this, and the effect of slowing light can be used to explain the long persistence of photoluminescence radiation $[10,11]$. Let us mark, that in these same areas of the spectrum it is possible to create the conditions for the spread of photons with "above light" speed and they form a cone of Vavilov-Tcherenkov radiation $[8,9]$.
If the frequency of the radiation $v$ higher than the natural frequency oscillators $v_{0 i}$, we face a situation, where the refractive index of the environment becomes less than unit: $n(v)<1$. The difference in the refractive index of the unit serves as a natural barrier to the spread of photons of light radiation, affects on the environment.

That obstacle can be overcome, assuming, that only part of photons incident on environment almost resonance radiation $\left(v \neq v_{0 \mathrm{i}}\right)$ will be utilize on dynamic compensation dispersion of the refractive index depending on the frequency for protect: $n(v) \rightarrow 1$. Populations lower and upper levels in the atoms of a two-level environment through the process (3) will be equalized and then we will be $n(v) \rightarrow 1$, which corresponds to enlightenment environment, while another portion of the photons of the same beam will be distributed through the medium without signs of slowing down, which tends to be happening in physical experiments.

Multiphoton mechanism of dynamic compensation of dispersion is well examplified by V. E. Ogluzdin [9] for the near-resonant propagation of light through atomic vapours potassium, as well as in the case application by V. E. Ogluzdin $[10,11]$ of this model to explain the phenomenon of photoluminescence.

Before turning directly to the interpretation RLS based on multiphoton model, we once again remind, that the spectral structure RLS radiation is usually three spectral component, and shifts Stockes and anti-Stockes components relative to the central, unbiased frequency component $v_{01}$ is not equal to each other. Typically, the intensity Stockes components RLS, according I. L. Fabelinskiy, exceeds intensity anti-Stockes components and in the spectrum of scattered radiation can abundant a continuum that extends to $100-150 \mathrm{~cm}^{-1}$ in both sides of the frequency of line of exciting radiation.

If the proposed model (see Equations (2) and (3)) is true, then this scenario suggests, that the emergence of Stokes and anti-Stockes components of RLS is associated with the excitement of independent sets of oscillator environment and its opening on the frequency $v=v_{01}$.

According to (3) Stokes and anti-Stockes components $v_{s}$, $v_{a s}$ of RLS and their corresponding Bohr jump-frequencies $v_{01}, v_{02} \ldots v_{0 i}$ are on different sides on the frequency of the exciting radiation $v$. Naturally, the emergence of Stokes and anti-Stockes components can only appear in an area occupied by light beam. Where excited radiation is lacking, properties of environment do not change.

Under the experiment, this will not preclude the distribution of new frequency components $v_{s}, v_{a s}$ across imperturbable environment and registering them at photoplate. Generally speaking, this radiation is dispersed into a $4 \pi$ steradian. Recall, that in the paper (I. L. Fabelinskiy 1957), the registration spectrum RLS realize in the transverse direction concerning direction of the incident radiation.

The frequency $v$ of incident radiation may by accident 
coincide with the frequency of the oscillator of environment $\left(v=v_{0 i}\right)$. Since the refractive index [2] in this case $n(v)=1$, the portion of the incident radiation at a frequency $v$ can easily pass through medium. But we must remember about of oscillators environment, Bohr jumpfrequencies of which are shifted to the frequency of the incident radiation $v$ in the blue and red regions of the spectrum. Ultimately, their presence, the dispersion characteristics of the refraction indexes therefore: $n(v)>$ 1 or $n(v)<1$ determine process RLS, according to the ratio of (3).

It is commonly supposed that RLS process is due microfluctuations density of the environment and the orientation of its species. But the self influence of the radiation to alter the optical properties and, in particular, the emergence of these fluctuations is not considered.

However, microfluctuations of the refractive index of the environment may arise from the fact, that the same radiation is acted on by the environment, could be a source of such microfluctuations and thus cause deviations (or diffraction), as the radiation and its of Stokes and anti-Stockes components, which is generally observed in such experiments.

Incidentally, it is understandable situation, which occurs in the case of the Stimulated (Mandelshtam-) Brillouin scattering (SBS), when the Stokes components of radiation can be traced in the opposite direction; they are reflected [13]. Really, in the moment of the generation of the inversion of oscillators of medium for frequencies $v$ $<v_{0 i}$ at the front pulse of light can creat conditions, protecting to a change in the refractive index of the environment: $n(v)<1$.

The inversion of a two-level environment produce in the region Stocks of frequencies $\left(v_{s}<v_{0 i}\right)$ reduction in the refractive index $n(v)$ of medium [14]. If will be realized condition $n\left(v_{s}\right)<1$, then for SBS component take place high reflectivity and this condition determine the opposite direction of its spread.

\section{Conclusions}

A agreement between own frequencies different oscillators of environment and Bohr jump-frequencies of this environment has brought to the interpretation of the fine structure RLS mechanism, on the one hand, based on the classical theory of dispersion, and on the other, based on the concept of quantum, the corpuscular nature of light.

The possibility of observing RLS a wide body angle ( $4 \pi$ steradian) testifies to the absence of significant barriers to its spread.

The intensity of anti-Stokes components observed in the discussed experiment is smaller, than Stokes items and depend from the refractive index of oscillators of environment, whose frequencies correspond to a frequency of the incident radiation. Reverse direction of the reflected Stokes SBS due to a modified dispersion dependence of oscillators of environment brought pumping radiation.

\section{References}

[1] I. L. Fabelinski, "Molecular scattering of light," Uspekhi Fizicheskikh Nauk, Russian Academy of Sciences, Moscow, in Russian, Vol. 63, 1957, p. 355.

[2] M. Born and E. Wolf, "Principles of Optics," Pergamon Press, Oxford, London, Edinburg, New York, Paris, Frankfurt, 1970.

[3] C. F. Bohren and D. R. Huffman, "Absorption and Scattering of Light by Small Particles," Willey, New York, 1983.

[4] N. Bohr, "Atomic Theory and the Description of Nature," Cambridge University Press, Cambridge, 1934.

[5] E. Fermi, "Notes on Quantum Mechanics: A Course Given by Enrico Fermi at the University of Chicago," University of Chicago Press, Chicago, 1965

[6] V. E. Ogluzdin, "The Role of Bohr Freguencies in the scattering, Luminescence, and Generation of Radiation in Different Media," Physics-Uspekhi, in English, Vol. 49, No. 4, 2006, p. 401; Uspekhi Fizicheskikh Nauk, Russian Academy of Sciences, Moscow, in Russian, Vol. 176, 2006, p. 415.

[7] M. Garbuny, "Optical Physics," Academic Press, New York and London, 1965.

[8] V. E. Ogluzdin, "Photons Traveling at the Speed of Light in Two-Level Atomic Medium as a Sourse of Cherenkov Radiation Cones," Physics-Uspekhi, in English, Vol. 47, 2004, p. 829; Uspekhi Fizicheskikh Nauk, Russian Academy of Sciences, Moscow, in Russian, Vol. 174, 2004, p. 895.

[9] V. E. Ogluzdin, "Vavilov-Cherenkov Effect under Conditions of Almost Resonant Interaction between Intense Light Beams and Atomic Potassium Vapor," Soviet Physics-Journal of Experimental and Teoretical Physics, in English, Vol. 52, 1980, p. 181; Zhurnal Eksperimentalnoi i Teoreticheskoi Fiziki, Moscow, in Russian, Vol. 79, 1980, p. 361.

[10] V. E. Ogluzdin, "Interpretation of the Visible Photoluminescence of Inequisized Silicon Nanoparticles Suspended in Ethanol," Semiconductors, in Eglish, Vol. 39, 2005, p. 884; Fizika i Tekhnika Poluprovodnikov, S. Petersburg, in Russian, Vol. 70, 2005, p. 920.

[11] V. E. Ogluzdin, "Bulletin of Russian Academy of Science," Physics, Vol. 3, 2006, p. 475.

[12] R. H. Pantell and H. E. Puthoff, "Fundamentals of Quantum Electronics," John Wiley \& Sons, New York, London, Sydney, Toronto, 1969.

[13] V. I. Bespalov, A. A. Kubarev and G. A. Pasmanik, "Stmulated Brillouin Scattering," Izv. Vysch. Uchebn. Zaved. Radiofiz., Vol. 13, 1970, p. 1433; Soviet Radiophysics, Gorkiy, in Russian.

[14] F. A. Korolev, "Theoretical Optics: An Introduction," in Russian, High School, Moscow, 1966, p. 298. 